Glasgow Math. J. 51 (2009) 425-440. (C) 2009 Glasgow Mathematical Journal Trust. doi:10.1017/S0017089509005084. Printed in the United Kingdom

\title{
ON WEAKLY RIGID RINGS
}

\author{
A. R. NASR-ISFAHANI and A. MOUSSAVI \\ Department of Mathematics, Tarbiat Modares University, PO Box 14115-175, Tehran, Iran \\ e-mail:a_nasr_isfahani@yahoo.com,moussavi_a5@yahoo.com
}

(Received 7 April 2008; revised 24 July 2008; accepted 20 November 2008)

\begin{abstract}
Let $R$ be a ring with a monomorphism $\alpha$ and an $\alpha$-derivation $\delta$. We introduce $(\alpha, \delta)$-weakly rigid rings which are a generalisation of $\alpha$-rigid rings and investigate their properties. Every prime ring $R$ is $(\alpha, \delta)$-weakly rigid for any automorphism $\alpha$ and $\alpha$-derivation $\delta$. It is proved that for any $n$, a $\operatorname{ring} R$ is $(\alpha, \delta)$-weakly rigid if and only if the $n$-by- $n$ upper triangular matrix $\operatorname{ring} T_{n}(R)$ is $(\bar{\alpha}, \bar{\delta})$-weakly rigid if and only if $M_{n}(R)$ is $(\bar{\alpha}, \bar{\delta})$-weakly rigid. Moreover, various classes of $(\alpha, \delta)$-weakly rigid rings is constructed, and several known results are extended. We show that for an $(\alpha, \delta)$-weakly rigid ring $R$, and the extensions $R[x], R[[x]], R[x ; \alpha, \delta], R\left[x, x^{-1} ; \alpha\right], R[[x ; \alpha]], R\left[\left[x, x^{-1} ; \alpha\right]\right]$, the ring $R$ is quasi-Baer if and only if the extension over $R$ is quasi-Baer. It is also proved that for an $(\alpha, \delta)$-weakly rigid ring $R$, if any one of the rings $R, R[x], R[x ; \alpha, \delta]$ and $R\left[x, x^{-1} ; \alpha\right]$ is left principally quasi-Baer, then so are the other three. Examples to illustrate and delimit the theory are provided.
\end{abstract}

AMS Subject Classification. 16S36. 16W60.

1. Introduction. Throughout this paper $R$ denotes an associative ring with unity; $\alpha$ is a monomorphism of $R$ which is not assumed to be surjective; and $\delta$ an $\alpha$-derivation of $R$, that is $\delta$ is an additive map such that $\delta(a b)=\delta(a) b+\alpha(a) \delta(b)$, for all $a, b \in R$.

According to Krempa [18], a monomorphism $\alpha$ of a ring $R$ is called to be rigid if $a \alpha(a)=0$ implies $a=0$ for $a \in R$. A ring $R$ is said to be $\alpha$-rigid if there exists a rigid monomorphism $\alpha$ of $R$.

The second author and E. Hashemi in [12] defined a ring $R$ with a monomorphism $\alpha$ and an $\alpha$-derivation $\delta$, to be called $(\alpha, \delta)$-compatible if for each $a, b \in R, a b=$ 0 implies $a \delta(b)=0$, and $a b=0$ if and only if $a \alpha(b)=0$.

We say a $\operatorname{ring} R$ with a monomorphism $\alpha$ and $\alpha$-derivation $\delta$, to be called $(\alpha, \delta)$ weakly rigid if for each $a, b \in R, a R b=0$ implies $a \delta(b)=0$, and $a R b=0$ if and only if $a \alpha(R b)=0$.

By [12], a ring $R$ is $\alpha$-rigid if and only if it is $(\alpha, \delta)$-compatible and reduced. Notice that the class of $\alpha$-rigid rings and $(\alpha, \delta)$-compatible rings is a narrow class of rings, and it is easy to see that every $(\alpha, \delta)$-compatible ring is $(\alpha, \delta)$-weakly rigid; but there are various classes of $(\alpha, \delta)$-weakly rigid rings which are not $(\alpha, \delta)$-compatible, as we will see in Section 2.

It is clear that every prime ring $R$ is $(\alpha, \delta)$-weakly rigid for any automorphism $\alpha$ and $\alpha$-derivation $\delta$. In this paper we prove that for any positive integer $n$, a ring $R$ is $(\alpha, \delta)$-weakly rigid if and only if the $n$-by- $n$ upper triangular matrix $\operatorname{ring} T_{n}(R)$ is $(\bar{\alpha}, \bar{\delta})$-weakly rigid if and only if the matrix $\operatorname{ring} M_{n}(R)$ is $(\bar{\alpha}, \bar{\delta})$-weakly rigid. 
We also show that if $R$ is a semiprime $(\alpha, \delta)$-weakly rigid ring, then the ring of polynomials $R[X]$, for $X$ an arbitrary non-empty set of indeterminates, is a semiprime $(\bar{\alpha}, \bar{\delta})$-weakly rigid ring. If $R$ is an $\alpha$-rigid ring, then $R[x] /\left\langle x^{n}\right\rangle$ is an $(\bar{\alpha}, \bar{\delta})$-weakly rigid ring, for any $n \geq 2$, where $\left\langle x^{n}\right\rangle$ is the ideal generated by $x^{n}$.

Suppose that $R$ is a ring with a monomorphism $\alpha$ and $\alpha$-derivation $\delta$. We show that when $R$ has a classical quotient ring $Q$ and $R$ is $(\alpha, \delta)$-weakly rigid, $Q$ is also $(\bar{\alpha}, \bar{\delta})$-weakly rigid.

Recall from $[9,17]$ that a ring $R$ is called (quasi-) Baer if the right annihilator of every (ideal) non-empty subset of $R$ is generated, as a right ideal, by an idempotent of $R$. Recall from [6] that a ring is called left (resp. right) principally quasi-Baer (or simply left (resp. right) p.q.-Baer) if the left annihilator of a principal left (resp. right) ideal of $R$ is generated by an idempotent.

Armendariz [1] has shown that a reduced ring $R$ (i.e. having no non-zero nilpotent elements) is Baer if and only if $R[x]$ is Baer.

The Ore extensions of quasi-Baer and p.q.-Baer rings have been investigated by many authors $[1,5,7,11,12,14,15,21,22,24]$. Most of these have worked either with the case $\delta=0$ and $\alpha$ an automorphism or with the case in which $\alpha$ is the identity.

Birkenmeier et al. in [5, Theorem 1.2] show that if $R$ is a quasi-Baer ring and $\alpha$ is an automorphism of $R$, then $R[x ; \alpha]$ is a quasi-Baer ring. They also provided an example of a quasi-Baer ring $R$ with an endomorphism $\alpha$ such that $R[x ; \alpha]$ is not quasi-Baer. In [7], they also proved that a ring $R$ is right p.q.-Baer if and only if the polynomial ring $R[x]$ is right p.q.-Baer.

Hong et al. in [14] have shown that if $R$ is an $\alpha$-rigid ring, then $R$ is Baer if and only if $R[x ; \alpha, \delta]$ is a Baer ring if and only if the skew power series ring $R[[x ; \alpha]]$ is a Baer ring. By [5, Lemma 1.9], a reduced (and hence $\alpha$-rigid) ring is Baer if and only if it is quasi-Baer, and by [14] a ring $R$ is $\alpha$-rigid if and only if the Ore extension $R[x ; \alpha, \delta]$ is reduced. The second author and E. Hashemi in [12], extended Hong et al.'s results of [14]. Note also that there is a commutative reduced p.q.-Baer ring which the power series ring is not a p.q.-Baer ring [20].

Although the class of $\alpha$-rigid (or $(\alpha, \delta)$-compatible) quasi-Baer rings is too narrow, we show that there are many rich classes of $(\alpha, \delta)$-weakly rigid quasi-Baer rings. For every prime ring $R$ and any automorphism $\alpha$ and $\alpha$-derivation $\delta$, the rings $M_{n}(R), T_{n}(R), R[X]$ and power series ring $R[[X]]$ are $(\bar{\alpha}, \bar{\delta})$-weakly rigid quasi-Baer rings.

For an $(\alpha, \delta)$-weakly rigid ring $R$, the relationship between $R$, the skew polynomial ring $R[x ; \alpha, \delta]$, skew Laurent polynomial ring $R\left[x ; x^{-1} ; \alpha\right]$, skew power series ring $R[[x ; \alpha]]$ and skew Laurent power series ring $R\left[\left[x ; x^{-1} ; \alpha\right]\right]$ is studied, and we show that strong connections exist between these rings and their various properties. Known results relating to $\alpha$-rigid rings can be obtained as corollaries of our results. Among applications, we show that a number of interesting properties of an $(\alpha, \delta)$-weakly rigid ring $R$ such as the quasi-Baer property and the principally quasi-Baer property transfer to its extensions and vice versa.

We provide examples which show that, in general, the quasi-Baerness (or p.q.Baerness) of $R$ and the aforementioned extensions do not depend on each other. As a consequence we extend and unify several known results.

2. Weakly rigid rings. In this section the notion of $(\alpha, \delta)$-weakly rigid rings is introduced, and a number of properties of this generalisation are established. We give a good supply of examples of $(\alpha, \delta)$-weakly rigid rings. 
For a non-empty subset $X$ of a ring $R, r_{R}(X)=\{c \in R \mid X c=0\}$ (respectively $\ell_{R}(X)=\{c \in R \mid c X=0\}$ ) is called the right (respectively left) annihilator of $X$ in $R$.

DEFINITION 2.1. A ring $R$ with a monomorphism $\alpha$, is called $\alpha$-weakly rigid if for each $a, b \in R, a R b=0$ if and only if $a \alpha(R b)=0$.

A ring $R$ with a derivation $\delta$ is called $\delta$-weakly rigid if for each $a, b \in R, a R b=$ 0 implies $a \delta(b)=0$.

A ring $R$ with a monomorphism $\alpha$ and $\alpha$-derivation $\delta$, is called $(\alpha, \delta)$-weakly rigid if it is both $\alpha$-weakly rigid and $\delta$-weakly rigid.

Every $\alpha$-compatible ring is $\alpha$-weakly rigid, and $(\alpha, \delta)$-compatible rings are clearly $(\alpha, \delta)$-weakly rigid; but there are various classes of $(\alpha, \delta)$-weakly rigid rings which are not $(\alpha, \delta)$-compatible (and hence not $\alpha$-rigid), as we will see in this section.

Let $R$ be a ring, $\alpha$ an endomorphism and $\delta$ an $\alpha$-derivation of $R$. It is easy to see that for any subring $S$ of the full matrix $\operatorname{ring} M_{n}(R), \bar{\alpha}: S \rightarrow S$, given by $\bar{\alpha}\left(\left(a_{i j}\right)\right)=\left(\alpha\left(a_{i j}\right)\right)$, is a homomorphism, and $\bar{\delta}: S \rightarrow S$, given by $\bar{\delta}\left(\left(a_{i j}\right)\right)=\left(\delta\left(a_{i j}\right)\right)$, is an $\bar{\alpha}$-derivation. We shall denote the $(i, j)$-th entry of a matrix $A \in M_{n}(R)$ by $A_{i j}$.

In the following example we see that for an $\alpha$-rigid ring $R, M_{n}(R)$ or $T_{n}(R)$ is not necessarily $\bar{\alpha}$-compatible (and hence not $\bar{\alpha}$-rigid).

EXAMPLE 2.2. Let $D$ be a domain and $\alpha$ be the automorphism of the polynomial ring $R:=D\left[x_{1}, x_{2}, \ldots, x_{m}\right]$, with indeterminates $x_{1}, x_{2}, \ldots, x_{m}$, given by $\alpha\left(x_{i}\right)=x_{i+1}$ for $1 \leq i \leq m-1$ and $\alpha\left(x_{m}\right)=x_{1}$. Then $R$ is an $\alpha$-rigid ring. Take $a=E_{11} x_{1}+E_{12} x_{2}$ and $b=E_{12} x_{2}-E_{22} x_{1}$, where $E_{i j}$ denotes the matrix unit. We have $a, b \in T_{n}(R) \subseteq$ $M_{n}(R)$. It is seen that $a b=0$ but $a \bar{\alpha}(b) \neq 0$. Hence neither $M_{n}(R)$ nor $T_{n}(R)$ is $(\bar{\alpha}, \bar{\delta})$ compatible.

Although the class of $\alpha$-rigid (or $(\alpha, \delta)$-compatible) rings do not pass to matrix rings by the above example, we show that the weakly rigid property overcomes these shortfalls.

THEOREM 2.3. Let $R$ be a ring and $\alpha$ an endomorphism of $R$. Then the following are equivalent:

(i) $R$ is an $\alpha$-weakly rigid ring;

(ii) $M_{n}(R)$ is an $\bar{\alpha}$-weakly rigid ring for every positive integer $n$;

(iii) $M_{n}(R)$ is an $\bar{\alpha}$-weakly rigid ring for some positive integer $n$.

Proof. (i) $\Rightarrow$ (ii). Suppose that $R$ is $\alpha$-weakly rigid and $A M_{n}(R) B=0$, with $A=\left(a_{i j}\right), B=\left(b_{i j}\right) \in M_{n}(R)$. To prove that $A \bar{\alpha}\left(M_{n}(R) B\right)=0$, it is enough to show that, for each $r \in R$ and $1 \leq i, j \leq n, A \bar{\alpha}\left(r E_{i j} B\right)=0$. To do this, we show that $\left(A \bar{\alpha}\left(r E_{i j} B\right)\right)_{t k}=0$, for each $1 \leq t, k \leq n$, where $\left(A \bar{\alpha}\left(r E_{i j} B\right)\right)_{t k}$ is the $(t, k)$-th entry of the matrix $A \bar{\alpha}\left(r E_{i j} B\right)$. Now we have $r E_{i j} B=r b_{j 1} E_{i 1}+r b_{j 2} E_{i 2}+\cdots+r b_{j n} E_{i n}$. So $\bar{\alpha}\left(r E_{i j} B\right)=\alpha\left(r b_{j 1}\right) E_{i 1}+\alpha\left(r b_{j 2}\right) E_{i 2}+\cdots+\alpha\left(r b_{j n}\right) E_{i n}$. Thus $\left(A \bar{\alpha}\left(r E_{i j} B\right)\right)_{t k}=\left(a_{t 1} E_{t 1}+\right.$ $\left.a_{t 2} E_{t 2}+\cdots+a_{t n} E_{t n}\right)\left(\alpha\left(r b_{j k}\right) E_{i k}\right)=a_{t i} \alpha\left(r b_{j k}\right) E_{t k}$. Therefore it is enough to show that $a_{t i} \alpha\left(r b_{j k}\right)=0$, for each $r \in R$ and $1 \leq i, j, t, k \leq n$. But we have $A M_{n}(R) B=0$, so $\left(A r E_{i j} B\right)_{t k}=0$, and hence $a_{t i} r b_{j k}=0$ for each $r \in R$ and $1 \leq i, j, t, k \leq n$. So $a_{t i} R b_{j k}=0$, and hence $a_{t i} \alpha\left(R b_{j k}\right)=0$, since $R$ is $\alpha$-weakly rigid. Thus $a_{t i} \alpha\left(r b_{j k}\right)=0$, for each $r \in R$ and $1 \leq i, j, t, k \leq n$, and hence $A \bar{\alpha}\left(M_{n}(R) B\right)=0$. Next assume that $A \bar{\alpha}\left(M_{n}(R) B\right)=0$, with $A=\left(a_{i j}\right), B=\left(b_{i j}\right) \in M_{n}(R)$. To prove that $A M_{n}(R) B=0$, it is enough to show that $A r E_{i j} B=0$, for each $r \in R$ and $1 \leq i, j \leq n$. To do this, we show that $\left(A r E_{i j} B\right)_{t k}=a_{t i} r b_{j k} E_{t k}=0$, for each $1 \leq t, k \leq n$. Since $A \bar{\alpha}\left(M_{n}(R) B\right)=0$, we get $\left(A \bar{\alpha}\left(r E_{i j} B\right)\right)_{t k}=0$, so $a_{t i} \alpha\left(r b_{j k}\right)=0$, for each $1 \leq i, j, t, k \leq n$. Thus $a_{t i} \alpha\left(R b_{j k}\right)=0$, and 
hence $a_{t i} R b_{j k}=0$ for each $1 \leq i, j, t, k \leq n$. Consequently $A M_{n}(R) B=0$, and $\operatorname{so} M_{n}(R)$ is $\bar{\alpha}$-weakly rigid. (ii) $\Rightarrow$ (iii). Is trivial.

(iii) $\Rightarrow$ (i). Suppose that for some $n, M_{n}(R)$ is an $\bar{\alpha}$-weakly rigid ring and that $a R b=0$ with $a, b \in R$. It is easy to see that $a E_{11} M_{n}(R) b E_{11}=0$, and hence $a E_{11} \bar{\alpha}\left(M_{n}(R) b E_{11}\right)=0$, since $M_{n}(R)$ is $\bar{\alpha}$-weakly rigid. So $a E_{11} \bar{\alpha}\left(r E_{11} b E_{11}\right)=0$, and hence $a \alpha(r b) E_{11}=0$ for each $r \in R$; consequently $a \alpha(R b)=0$. Next assume that $a \alpha(R b)=0$, so $a E_{11} \bar{\alpha}\left(M_{n}(R) b E_{11}\right)=0$. Thus $a E_{11} M_{n}(R) b E_{11}=0$, since $M_{n}(R)$ is $\bar{\alpha}-$ weakly rigid. Therefore for each $r \in R, a E_{11} r E_{11} b E_{11}=0$ so $a R b=0$, whence $R$ is $\alpha$-weakly rigid.

THEOREM 2.4. Let $R$ be a ring and $\delta$ a derivation of $R$. Then the following are equivalent:

(i) $R$ is a $\delta$-weakly rigid ring;

(ii) $M_{n}(R)$ is a $\bar{\delta}$-weakly rigid ring for every positive integer $n$;

(iii) $M_{n}(R)$ is an $\bar{\delta}$-weakly rigid ring for some positive integer $n$.

Proof. (i) $\Rightarrow$ (ii). Suppose that $R$ is $\delta$-weakly rigid and $A M_{n}(R) B=0$, with $A=$ $\left(a_{i j}\right), B=\left(b_{i j}\right) \in M_{n}(R)$. To prove that $A \bar{\delta}(B)=0$, it is enough to show that, for each $1 \leq i, j \leq n,(A \bar{\delta}(B))_{i j}=0$. Now for each $1 \leq i, j \leq n$, we have $(A \bar{\delta}(B))_{i j}=\left(a_{i 1} \delta\left(b_{1 j}\right)+\right.$ $\left.a_{i 2} \delta\left(b_{2 j}\right)+\cdots+a_{i n} \delta\left(b_{n j}\right)\right) E_{i j}$. Since $A M_{n}(R) B=0$, we get $A r E_{t t} B=0$ for each $r \in R$ and $1 \leq t \leq n$. So $\left(A r E_{t t} B\right)_{i j}=0$ for each $1 \leq i, j \leq n$. But $\left(A r E_{t t} B\right)_{i j}=a_{i t} r b_{t j} E_{i j}$, so $a_{i t} r b_{t j}=0$ for each $r \in R$ and $1 \leq i, j, t \leq n$. Thus $a_{i t} \delta\left(b_{t j}\right)=0$, so $(A \bar{\delta}(B))_{i j}=0$, and hence $M_{n}(R)$ is $\bar{\delta}$-weakly rigid.

(ii) $\Rightarrow$ (iii). Is trivial.

(iii) $\Rightarrow$ (i). Assume that $M_{n}(R)$ is $\bar{\delta}$-weakly rigid for some $n$, and $a R b=0$ with $a, b \in R$. Then we have $a E_{11} M_{n}(R) b E_{11}=0$, and since $M_{n}(R)$ is $\bar{\delta}$-weakly rigid, $a E_{11} \bar{\delta}\left(b E_{11}\right)=0$. Thus $a \delta(b) E_{11}=0$, consequently $R$ is $\delta$-weakly rigid.

COROLlaRY 2.5. For any positive integer $n$, a ring $R$ is $(\alpha, \delta)$-weakly rigid if and only if $M_{n}(R)$ is an $(\bar{\alpha}, \bar{\delta})$-weakly rigid ring.

THEOREM 2.6. Let $R$ be a ring and $\alpha$ an endomorphism of $R$. Then the following are equivalent:

(i) $R$ is an $\alpha$-weakly rigid ring;

(ii) $T_{n}(R)$ is an $\bar{\alpha}$-weakly rigid ring for every positive integer $n$;

(iii) $T_{n}(R)$ is an $\bar{\alpha}$-weakly rigid ring for some positive integer $n$.

Proof. The proof is similar to that of Theorem 2.3.

THEOREM 2.7. Let $R$ be a ring and $\delta$ a derivation of $R$. Then the following are equivalent:

(i) $R$ is a $\delta$-weakly rigid ring;

(ii) $T_{n}(R)$ is a $\bar{\delta}$-weakly rigid ring for every positive integer $n$;

(iii) $T_{n}(R)$ is a $\bar{\delta}$-weakly rigid ring for some positive integer $n$.

Proof. The proof is similar to that of Theorem 2.4.

EXAMPLE 2.8. Let $D$ be a prime ring and $\alpha$ be the automorphism of the polynomial $\operatorname{ring} R:=D\left[x_{1}, x_{2}, \ldots, x_{m}\right]$, with indeterminates $x_{1}, x_{2}, \ldots, x_{m}$, given by $\alpha\left(x_{i}\right)=x_{i+1}$ for $1 \leq i \leq m-1$ and $\alpha\left(x_{m}\right)=x_{1}$. Then $R$ is an $\alpha$-weakly rigid ring. As in Example 2.2 , it is seen that neither $M_{n}(R)$ nor $T_{n}(R)$ is $(\bar{\alpha}, \bar{\delta})$-compatible. However by Theorems 2.3 and $2.6, M_{n}(R)$ and $T_{n}(R)$ are both $\bar{\alpha}$-weakly rigid rings. 
Let $R$ be a ring. Define $R_{n}=R I_{n}+\sum_{i=1}^{n} \sum_{j=i+1}^{n} R E_{i j}$, for $n \geq 2$, where $E_{i j}$ is the matrix units for all $i, j$ and $I_{n}$ is the identity matrix. Note that $R_{n}$ is a subring of $T_{n}(R)$.

THEOREM 2.9. If $R$ is an $\alpha$-rigid ring, then $R_{n}$ is an $(\bar{\alpha}, \bar{\delta})$-weakly rigid ring, for any $n$.

Proof. Assume $A, B \in R_{n}$ and $A R_{n} B=0$. Since $R_{n}=R I_{n}+\sum_{i=1}^{n} \sum_{j=i+1}^{n} R E_{i j}$, in order to prove that $A \bar{\alpha}\left(R_{n} B\right)=0$, it is enough to show that $A \bar{\alpha}\left(r I_{n} B\right)=A \bar{\alpha}\left(r E_{i j} B\right)=0$, for each $r \in R$ and $1 \leq i \leq n, i+1 \leq j \leq n$. First suppose that $r \in R$ and $1 \leq i \leq n$, $i+1 \leq j \leq n$; then $\left(A \bar{\alpha}\left(r E_{i j} B\right)\right)_{t k}=a_{t i} \alpha\left(r b_{j k}\right) E_{t k}$. Since $A R_{n} B=0,\left(A r E_{i j} B\right)_{t k}=0$, so $a_{t i} r b_{j k}=0$, and hence $a_{t i} R b_{j k}=0$. Since $R$ is $\alpha$-weakly rigid, $a_{t i} \alpha\left(R b_{j k}\right)=0$, so $\left(A \bar{\alpha}\left(r E_{i j} B\right)\right)_{t k}=0$; hence $A \bar{\alpha}\left(r E_{i j} B\right)=0$. Now, for each $1 \leq i \leq j \leq n,\left(A \bar{\alpha}\left(r I_{n} B\right)\right)_{i j}=$ $a_{i i} \alpha(r) \alpha\left(b_{i j}\right) E_{i j}+a_{i, i+1} \alpha(r) \alpha\left(b_{i+1, j}\right) E_{i j}+\cdots+a_{i j} \alpha(r) \alpha\left(b_{j j}\right) E_{i j}$. Since $A R_{n} B=0$, we get $\operatorname{ArI}_{n} B=0$. Hence $\left(A r I_{n} B\right)_{i j}=a_{i i} r b_{i j} E_{i j}+a_{i, i+1} r b_{i+1, j} E_{i j}+\cdots+a_{i j} r b_{j j} E_{i j}=0 .(*)$ Since $\left(A E_{i j} B\right)_{i j}=0, a_{i i} b_{j j}=0$; and since $\left(A E_{i+1, j} B\right)_{i j}=0, a_{i, i+1} b_{j j}=0$. By this way, after $j-i$ steps, we get $a_{i, j-1} b_{j j}=0$, since $\left(A E_{j-1, j} B\right)_{i j}=0$. We have $a_{i i} b_{j j}=a_{i, i+1} b_{j j}=$ $\cdots=a_{i, j-1} b_{j j}=0$. Since $R$ is $\alpha$-rigid, it is reduced, $b_{j j} a_{i i}=b_{j j} a_{i, i+1}=\cdots=b_{j j} a_{i, j-1}=$ 0 . So by multiplying $(*)$ from left by $b_{j j}$ we get $b_{j j} a_{i j} r b_{j j}=0$. Thus $a_{i j} r b_{j j}=0$, since $R$ is reduced. Hence $a_{i j} R b_{j j}=0$, and since $R$ is $\alpha$-rigid, $a_{i j} \alpha\left(R b_{j j}\right)=0$. We have $\left(A E_{i, j-1} B\right)_{i j}=0$, so $a_{i i} b_{j-1, j} E_{i j}=0$. Also $\left(A E_{i+1, j-1} B\right)_{i, j}=0$, so $a_{i, i+1} b_{j-1, j} E_{i j}=0$. Continuing in this way $\left(A E_{j-2, j-1} B\right)_{i j}=0$, so $a_{i, j-2} b_{j-1, j}=0$. By multiplying $(*)$ from left by $b_{j-1, j}$ we get $b_{j-1, j} a_{i, j-1} r b_{j-1, j}=0$, which implies $a_{i, j-1} R b_{j-1, j}=0$, and hence $a_{i, j-1} \alpha\left(R b_{j-1, j}\right)=0$, since $R$ is $\alpha$-rigid. By this way, after $j-i$ steps, we get $\left(A \bar{\alpha}\left(r I_{n} B\right)\right)_{i j}=0$, so $A \bar{\alpha}\left(r I_{n} B\right)=0$, and hence $A \bar{\alpha}\left(R_{n} B\right)=0$. Now suppose that $A \bar{\alpha}\left(R_{n} B\right)=0$. By a similar method as employed in the above argument we can show that $A R_{n} B=0$, and hence $R_{n}$ is a $\bar{\alpha}$-weakly rigid. Next assume that $A R_{n} B=0$; we then show that $A \bar{\delta}(B)=0$. For each $1 \leq i \leq j \leq n, \quad(A \bar{\delta}(B))_{i j}=a_{i i} \delta\left(b_{i j}\right) E_{i j}+$ $a_{i, i+1} \delta\left(b_{i+1, j}\right) E_{i j}+\cdots+a_{i j} \delta\left(b_{j j}\right) E_{i j}$. Since $A R_{n} B=0, \operatorname{Ar}_{n} B=0$, so $\left(\operatorname{Ar} I_{n} B\right)_{i, j}=0$, and hence $a_{i i} r b_{i j} E_{i j}+a_{i, i+1} r b_{i+1, j} E_{i j}+\cdots+a_{i j} r b_{j j} E_{i j}=0$. As we have seen in the first part of the proof, $a_{i i} r b_{i j}=0, a_{i, i+1} r b_{i+1, j}=0, \cdots, a_{i j} r b_{j j}=0$. Since $R$ is $\alpha$-rigid, $a_{i i} \delta\left(b_{i j}\right)=0, a_{i, i+1} \delta\left(b_{i+1, j}\right)=0, \cdots, a_{i j} \delta\left(b_{j j}\right)=0$. This implies that $(A \bar{\delta}(B))_{i j}=0$ for each $i, j$, and the result follows.

In [19], T. K. Lee and Y. Zhou defined $V_{n}=\sum_{i=1}^{n-1} E_{i, i+1}$, for $n \geq 2$, where $E_{i j}$ is the matrix units for all $i, j$.

For even integers $n=2 k \geq 2, A_{n}^{e}(R):=\sum_{i=1}^{k} \sum_{j=k+i}^{n} R E_{i j}$, so we have $A_{n}(R)=$ $R I_{n}+R V_{n}+R V_{n}^{2}+\cdots+R V_{n}^{k-1}+A_{n}^{e}(R)$.

For odd integers $n=2 k+1 \geq 3, A_{n}^{o}(R):=\sum_{i=1}^{k+1} \sum_{j=k+i}^{n} R E_{i j}$, so we have $A_{n}(R)=$ $R I_{n}+R V_{n}+R V_{n}^{2}+\cdots+R V_{n}^{k-1}+A_{n}^{o}(R)$.

Note that $A_{n}(R)$ is a subring of $T_{n}(R)$.

THEOREM 2.10. If $R$ is an $\alpha$-rigid ring, then $A_{n}(R)$ is an $(\bar{\alpha}, \bar{\delta})$-weakly rigid ring, for any $n \geq 2$.

Proof. The proof is similar to that of Theorem 2.9.

The trivial extension of $R$, which is denoted by $T(R, R)=\left\{\left(\begin{array}{ll}a & b \\ 0 & a\end{array}\right) \mid a, b \in R\right\}$, is a ring with matrix addition and multiplication. By Theorem 2.9, we see that if $R$ is $\alpha$-rigid, then $T(R, R)$ is $(\bar{\alpha}, \bar{\delta})$-weakly rigid. 
Let $R$ be a ring, and let

$$
T(R, n):=\left\{\left(\begin{array}{ccccc}
a_{1} & a_{2} & a_{3} & \cdots & a_{n} \\
0 & a_{1} & a_{2} & \cdots & a_{n-1} \\
0 & 0 & a_{1} & \cdots & a_{n-2} \\
\vdots & \vdots & \vdots & \ddots & \vdots \\
0 & 0 & 0 & \cdots & a_{1}
\end{array}\right) \mid a_{i} \in R\right\},
$$

with $n \geq 2$. Then $T(R, n)$ is a subring of the triangular matrix $\operatorname{ring} T_{n}(R)$.

Observe that $T(R, n) \cong R[x] /\left\langle x^{n}\right\rangle$, for any $n \geq 2$. A proof similar to that of Theorem 2.9, can be employed to prove that when $R$ is an $\alpha$-rigid $\operatorname{ring}, T(R, n)$, and hence $R[x] /\left\langle x^{n}\right\rangle$ is an $(\bar{\alpha}, \bar{\delta})$-weakly rigid ring, for any $n \geq 2$.

Let $R$ be a ring, $\alpha$ an endomorphism and $\delta$ an $\alpha$-derivation of $R$. Then $\bar{\alpha}: R[x] \rightarrow R[x]$, given by $\bar{\alpha}\left(\sum_{i=0}^{n} a_{i} x^{i}\right)=\sum_{i=0}^{n} \alpha\left(a_{i}\right) x^{i}$, and $\bar{\delta}: R[x] \rightarrow R[x]$, given by $\bar{\delta}\left(\sum_{i=0}^{n} a_{i} x^{i}\right)=\sum_{i=0}^{n} \delta\left(a_{i}\right) x^{i}$. Then $\bar{\alpha}$ is an endomorphism and $\bar{\delta}$ is an $\bar{\alpha}$-derivation of $R[x]$.

According to Hirano [13], a ring $R$ is called quasi-Armendariz if for polynomials $f(x)=a_{0}+a_{1} x+\cdots+a_{n} x^{n}, g(x)=b_{0}+b_{1} x+\cdots+b_{m} x^{m} \in R[x], f(x) R[x] g(x)=0$ if and only if $a_{i} R b_{j}=0$ for each $i, j$. In [13], Hirano studied some properties of quasi-Armendariz rings and proved that the condition quasi-Armendariz is a Morita invariant property and that every semiprime ring is quasi-Armendariz.

In [13, Theorem 3.16] Hirano shows that quasi-Armendariz conditions preserves by polynomial rings. Now we get the following:

THEOREM 2.11. If $R$ is a quasi-Armendariz $(\alpha, \delta)$-weakly rigid ring, then $R[x]$ is a quasi-Armendariz $(\bar{\alpha}, \bar{\delta})$-weakly rigid ring.

Since semiprime rings are quasi-Armendariz by [13], we get the following:

COROLLARY 2.12. If $R$ is a semiprime $(\alpha, \delta)$-weakly rigid ring, then $R[x]$ is a semiprime $(\bar{\alpha}, \bar{\delta})$-weakly rigid ring.

Recall that an idempotent $e \in R$ is left (respectively right) semi-central in $R$ if $R e=e R e($ respectively $e R=e R e)$.

THEOREM 2.13. If $R$ is an $(\alpha, \delta)$-weakly rigid ring and e is a left semi-central idempotent of $R$, then $e R, R$ and eRe are also $(\alpha, \delta)$-weakly rigid rings.

Proof. Suppose that er, es $\in e R$ and ereRes $=0$. Since $R e=e R e$, we have $e r R e s=$ 0 , so $\operatorname{er} \alpha(\operatorname{Res})=0, \operatorname{er} \delta(e s)=0$, and hence $\operatorname{er} \alpha(e \operatorname{Res})=0$, as $R$ is $(\alpha, \delta)$-weakly rigid. Now, if $\operatorname{er} \alpha(e \operatorname{Res})=0$, then $\operatorname{er} \alpha(\operatorname{Res})=0$, and hence erRes $=0$. Thus ereRes $=0$, and the result follows.

Notice that in Theorem 2.13, in order to restrict $\alpha, \delta$ from $R$ to $e R$, we need to assume that $\alpha(e), \delta(e) \in e R$, and this condition is satisfied for semi-central idempotents, since $R$ is $(\alpha, \delta)$-weakly rigid.

We now show that there exists an example of a ring $R$ with an idempotent $e \in R$ and an endomorphism $\alpha: R \rightarrow R$ such that $\alpha(e) \notin e R$, so the condition semi-central in Theorem 2.13 is not superfluous. 
EXAMPLE 2.14. Let $\mathbb{Q}$ be the ring of rational numbers; then $M_{2}(\mathbb{Q})$ is a prime ring. Let $\alpha$ be the automorphism of $M_{2}(\mathbb{Q})$, given by $\alpha\left(\left(\begin{array}{ll}a & b \\ c & d\end{array}\right)\right)=\left(\begin{array}{ll}1 & 1 \\ 0 & 1\end{array}\right)\left(\begin{array}{ll}a & b \\ c & d\end{array}\right)\left(\begin{array}{cc}1 & -1 \\ 0 & 1\end{array}\right)$, for each $a, b, c, d \in \mathbb{Q}$. Since $M_{2}(\mathbb{Q})$ is a prime ring and $\alpha$ an automorphism, $M_{2}(\mathbb{Q})$ is an $(\alpha, \delta)$-weakly rigid ring. Now $e=\left(\begin{array}{ll}0 & 0 \\ 1 & 1\end{array}\right)$ is an idempotent. We have $\alpha(e)=\left(\begin{array}{ll}1 & 0 \\ 1 & 0\end{array}\right)$, and $e \alpha(e)=\left(\begin{array}{ll}0 & 0 \\ 2 & 0\end{array}\right) \neq \alpha(e)$.

For an index set $I$, suppose that for each $i \in I, R_{i}$ is a ring, $\alpha_{i}: R_{i} \rightarrow R_{i}$ an endomorphism and $\delta_{i}: R_{i} \rightarrow R_{i}$ an $\alpha_{i}$-derivation of $R_{i}$. So $\bar{\alpha}: \prod_{i \in I} R_{i} \rightarrow \prod_{i \in I} R_{i}$ given by $\bar{\alpha}\left(\left\{r_{i}\right\}_{i \in I}\right)=\left\{\alpha_{i}\left(r_{i}\right)\right\}_{i \in I}$ is an endomorphism, and $\bar{\delta}: \prod_{i \in I} R_{i} \rightarrow \prod_{i \in I} R_{i}$ given by $\bar{\delta}\left(\left\{r_{i}\right\}_{i \in I}\right)=\left\{\delta_{i}\left(r_{i}\right)\right\}_{i \in I}$ is an $\bar{\alpha}$-derivation of $\prod_{i \in I} R_{i}$.

It is easy to see that if for each $i \in I, R_{i}$ is an $\left(\alpha_{i}, \delta_{i}\right)$ weakly rigid ring, then $\prod_{i \in I} R_{i}$ is also an $(\bar{\alpha}, \bar{\delta})$-weakly rigid ring.

Now we concern the classical quotient rings of $(\alpha, \delta)$-weakly rigid rings. A ring $R$ is called right Ore if given $a, b \in R$ with $b$ regular there exist $a_{1}, b_{1} \in R$ with $b_{1}$ regular such that $a b_{1}=b a_{1}$. It is a well-known fact that $R$ is a right Ore ring if and only if there exists the classical right quotient ring of $R$.

Let $R$ be an Ore ring, $\alpha$ an endomorphism and $\delta$ an $\alpha$-derivation of $R$. Define $\tilde{\alpha}: Q \rightarrow Q$ given by $\tilde{\alpha}\left(r c^{-1}\right)=\alpha(r) \alpha(c)^{-1}$ and $\tilde{\delta}: Q \rightarrow Q$ given by $\tilde{\delta}\left(r c^{-1}\right)=(\delta(r)-$ $\left.r c^{-1} \delta(c)\right) \alpha(c)^{-1}$. Then $\tilde{\alpha}$ is an endomorphism and $\tilde{\delta}$ an $\tilde{\alpha}$-derivation of the classical quotient ring $Q$ of $R$. The set of regular elements of $R$ is denoted by $\mathcal{C}$.

THEOREM 2.15. Suppose that there exists the classical quotient ring $Q$ of a ring $R$ with an endomorphism $\alpha$ and $\alpha$-derivation $\delta$. If $R$ is $(\alpha, \delta)$-weakly rigid, then $Q$ is $(\tilde{\alpha}, \tilde{\delta})$ weakly rigid.

Proof. Assume that $a Q b=0$, with $a=r_{1} c_{1}^{-1}, b=r_{2} c_{2}^{-1}$ for some $r_{1}, r_{2} \in R$ and $c_{1}, c_{2} \in \mathcal{C}$. We first show that $a \tilde{\alpha}(Q b)=0$. For each $r_{3} c_{3}^{-1} \in Q$, it is enough to show that $r_{1} c_{1}^{-1} \alpha\left(r_{3}\right) \alpha\left(c_{3}\right)^{-1} \alpha\left(r_{2}\right) \alpha\left(c_{2}\right)^{-1}=0$. Since $R$ satisfies the Ore condition, there exist $r_{4} \in R, c_{4} \in \mathcal{C}$ such that $r_{3} c_{3}^{-1}=c_{4}^{-1} r_{4}$. Hence $r_{1} c_{1}^{-1} \alpha\left(r_{3}\right) \alpha\left(c_{3}\right)^{-1} \alpha\left(r_{2}\right) \alpha\left(c_{2}\right)^{-1}=$ $r_{1} c_{1}^{-1} \alpha\left(c_{4}\right)^{-1} \alpha\left(r_{4}\right) \alpha\left(r_{2}\right) \alpha\left(c_{2}\right)^{-1}$. Since $R$ satisfies the Ore condition, there exist $r_{5} \in$ $R, c_{5} \in \mathcal{C}$ such that $r_{1}\left(\alpha\left(c_{4}\right) c_{1}\right)^{-1}=c_{5}^{-1} r_{5}$. Hence $r_{1} c_{1}^{-1} \alpha\left(r_{3}\right) \alpha\left(c_{3}\right)^{-1} \alpha\left(r_{2}\right) \alpha\left(c_{2}\right)^{-1}=$ $c_{5}^{-1} r_{5} \alpha\left(r_{4}\right) \alpha\left(r_{2}\right) \alpha\left(c_{2}\right)^{-1}$. Therefore it is enough to show that $r_{5} \alpha\left(r_{4}\right) \alpha\left(r_{2}\right) \alpha\left(c_{2}\right)^{-1}=0$. Since $r_{1} c_{1}^{-1} Q r_{2}=0$ and $\alpha\left(c_{4}\right)^{-1} Q r_{4} \subseteq Q$, we have $r_{1} c_{1}^{-1} \alpha\left(c_{4}\right)^{-1} Q r_{4} r_{2}=0$, and hence $c_{5}^{-1} r_{5} Q r_{4} r_{2}=0$. Thus $r_{5} Q r_{4} r_{2}=0$ so $r_{5} R r_{4} r_{2}=0$, and since $R$ is $(\alpha, \delta)$-weakly rigid, $r_{5} \alpha\left(R r_{4} r_{2}\right)=0$. So $r_{5} \alpha\left(r_{4}\right) \alpha\left(r_{2}\right)=0$; therefore $a \tilde{\alpha}(Q b)=0$.

Now we show that $a \tilde{\delta}(b)=0$. We have $a \tilde{\delta}(b)=r_{1} c_{1}^{-1}\left(\delta\left(r_{2}\right)-r_{2} c_{2}^{-1} \delta\left(c_{2}\right)\right) \alpha\left(c_{2}\right)^{-1}$. Since $r_{1} c_{1}^{-1} Q r_{2}=0$, we have $r_{1} c_{1}^{-1} r_{2} c_{2}^{-1} \delta\left(c_{2}\right) \alpha\left(c_{2}\right)^{-1}=0$. So it is enough to show that $r_{1} c_{1}^{-1} \delta\left(r_{2}\right)=0$. Since $R$ satisfies the Ore condition, there exist $r_{3} \in R, c_{3} \in \mathcal{C}$ such that $r_{1} c_{1}^{-1}=c_{3}^{-1} r_{3}$. So it is enough to show that $r_{3} \delta\left(r_{2}\right)=0$. Since $r_{1} c_{1}^{-1} Q r_{2}=0$, we have $c_{3}^{-1} r_{3} Q r_{2}=0$, so $r_{3} R r_{2}=0$. Since $R$ is $(\alpha, \delta)$-weakly rigid, $r_{3} \delta\left(r_{2}\right)=0$; therefore $a \tilde{\delta}(b)=0$.

Next suppose that $a \tilde{\alpha}(Q b)=0$, with $a=r_{1} c_{1}^{-1}, b=r_{2} c_{2}^{-1}$ for some $r_{1}, r_{2} \in R$ and $c_{1}, c_{2} \in \mathcal{C}$; we then show that $a Q b=0$. For each $r_{3} c_{3}^{-1} \in Q$, it is enough to show that $r_{1} c_{1}^{-1} r_{3} c_{3}^{-1} r_{2} c_{2}^{-1}=0$. Since $R$ satisfies the Ore condition, there exist $r_{4}, r_{5} \in R, c_{4}, c_{5} \in \mathcal{C}$ such that $r_{1} c_{1}^{-1}=c_{4}^{-1} r_{4}$ and $c_{3}^{-1} r_{2}=r_{5} c_{5}^{-1}$. So it is enough to show that $r_{4} r_{3} r_{5}=0$. Since $a \tilde{\alpha}(Q b)=0, c_{4}^{-1} r_{4} \tilde{\alpha}\left(R r_{3} c_{3}^{-1} r_{2} c_{2}^{-1}\right)=0$; so $r_{4} \alpha\left(R r_{3} r_{5}\right)=0$. Since $R$ is $(\alpha, \delta)$-weakly rigid, $r_{4} R r_{3} r_{5}=0$, and the result follows. 
3. Ore extensions of weakly rigid rings. The study of Baer rings has its roots in functional analysis [2, 17]. In [17] Kaplansky introduced Baer rings to abstract various properties of von Neumann algebras and complete regular $C^{*}$-rings. In [9] Clark defined a quasi-Baer ring and used it to characterise when a finite-dimensional algebra with unity over an algebraically closed field is isomorphic to a twisted matrix units semigroup algebra. Every prime ring is a quasi-Baer ring. Further examples of quasi-Baer rings are the Martindale right ring of quotients of any semiprime ring [8] and any semiprime right FPF ring [10, p. 168]. The class of quasi-Baer rings is closed under $n \times n$ upper triangular matrix rings and Morita invariance by [23].

A ring $R$ is called a right (respectively left) p.p.-ring if the right (respectively left) annihilator of an element of $R$ is generated by an idempotent; $R$ is called a p.p.-ring if it is both a right and a left p.p.-ring. The class of p.q.-Baer rings includes all biregular rings, all quasi-Baer rings and all abelian (i.e. every idempotent is central) p.p.-rings and is closed under direct products and Morita invariance. Further work has appeared in $[3-9,13,23]$.

The class of $\alpha$-rigid (or $(\alpha, \delta)$-compatible) quasi-Baer (or left p.q.-Baer) rings does not contain the class of prime rings, because prime rings are not necessarily reduced, and is not closed under extensions to matrix rings or triangular matrix rings, by Example 2.2. However the notion of an $(\alpha, \delta)$-weakly rigid quasi-Baer (or p.q.-Baer) ring overcomes these shortfalls.

For every prime ring $R$ and any automorphism $\alpha$ and $\alpha$-derivation $\delta$, the rings $M_{n}(R), T_{n}(R)$ and $R[X]$ and the power series ring $R[[X]]$ are quasi-Baer $(\alpha, \delta)$-weakly rigid rings.

We denote $R[x ; \alpha, \delta]$ the Ore extension whose elements are the polynomials $\sum_{i=0}^{n} r_{i} x^{i} \in R, r_{i} \in R$, where the addition is defined as usual and the multiplication by $x b=\alpha(b) x+\delta(b)$ for any $b \in R$.

The skew Laurent polynomial ring $R\left[x, x^{-1} ; \alpha\right]$, whose elements are finite sums of elements of the form $x^{-j} r x^{i}$, where $r \in R$ and $i, j$ are non-negative integers. Multiplication is subject to $x r=\alpha(r) x$ for all $r \in R$.

We also denote $R[[x ; \alpha]]$ the skew power series ring, whose elements are power series of the form $\sum_{i=0}^{\infty} r_{i} x^{i}$ with coefficients $r_{i} \in R$, where the addition is defined as usual and the multiplication subject to the condition $x b=\alpha(b) x$, for any $b \in R$. The set $\left\{x^{i}\right\}_{i \geq 0}$ is an Ore subset of $R[[x ; \alpha]]$, so that one can localise $R[[x ; \alpha]]$ and form the skew Laurent series ring $R\left[\left[x, x^{-1} ; \alpha\right]\right]$. Elements of $R\left[\left[x, x^{-1} ; \alpha\right]\right]$ are formal combinations of elements of the form $x^{-j} r x^{i}$, where $r \in R$ and $i, j$ are non-negative integers.

In this section we consider the relationship between the properties of being quasi-Baer and left p.q.-Baer of a ring $R$ and of the rings $R[x ; \alpha, \delta], R\left[x, x^{-1} ; \alpha\right]$, $R[[x ; \alpha]]$, and $R\left[\left[x, x^{-1} ; \alpha\right]\right]$, respectively.

We provide examples which show that, in general, the quasi-Baerness (or p.q.Baerness) of $R$ and the aforementioned extensions do not depend on each other.

We will begin by considering some properties of $(\alpha, \delta)$-weakly rigid rings.

Lemma 3.1. Suppose that $R$ is an $\alpha$-weakly rigid ring; then for each $a, b \in R$ and positive integers $i, j, a R b=0$ if and only if $\alpha^{i}(a) R \alpha^{j}(b)=0$.

Proof. Suppose that $a R b=0$, so $\alpha^{i}(a R b)=0$, and hence $\alpha^{i}(a) \alpha^{i}(R b)=0$. Since $R$ is $\alpha$-weakly rigid, $\alpha^{i}(a) R b=0$. So for each $r \in R, \alpha^{i}(a) r R b=0$; hence $\alpha^{i}(a) r \alpha^{j}(R b)=$ 0 . Thus $\alpha^{i}(a) r \alpha^{j}(b)=0$ whence $\alpha^{i}(a) R \alpha^{j}(b)=0$, for each $i, j$. Now assume that $\alpha^{i}(a) R \alpha^{j}(b)=0$, for each $i, j$. Since $R$ is $\alpha$-weakly rigid, $\alpha^{i}(a) \alpha^{i}\left(R \alpha^{j}(b)\right)=0$, so 
$\alpha^{i}\left(a R \alpha^{j}(b)\right)=0$. Since $\alpha$ is injective, $a R \alpha^{j}(b)=0$. So $a \alpha^{j}(R b)=0$, since $R$ is $\alpha$-weakly rigid $a R b=0$, and the result follows.

LemMA 3.2. Suppose that $R$ is $(\alpha, \delta)$-weakly rigid; then for each $a, b \in R$ and positive integers $i, j, a R b=0$ implies $\delta^{i}(a) R \delta^{j}(b)=0$.

Proof. Suppose that $a R b=0$, so for each $r \in R, a r R b=0$. Since $R$ is $(\alpha, \delta)$-weakly rigid, $\operatorname{ar} \delta^{j}(b)=0$ for each $r \in R$, and hence $a R \delta^{j}(b)=0$ for each positive integer $j$. Now we show by induction that for each positive integer $i, \delta^{i}(a) R \delta^{j}(b)=0$. For $i=1$, since $a R \delta^{j}(b)=0$, for each $r \in R, \delta\left(a r \delta^{j}(b)\right)=0$ so $\delta(a) r \delta^{j}(b)+\alpha(a) \delta\left(r \delta^{j}(b)\right)=0$. Since $a R \delta^{j}(b)=0$, we get $\alpha(a) R \delta^{j}(b)=0$ by Lemma 3.1. So $\alpha(a) R r \delta^{j}(b)=0$ for each $r \in R$. Since $R$ is $(\alpha, \delta)$-weakly rigid, $\alpha(a) \delta\left(r \delta^{j}(b)\right)=0$, so $\delta(a) r \delta^{j}(b)=0$. Now assume that the result is true for each $t<i$. So we have $\delta^{i-1}(a) R \delta^{j}(b)=0$. Hence $\delta\left(\delta^{i-1}(a) r \delta^{j}(b)\right)=0$ for each $r \in R$. So $\delta^{i}(a) r \delta^{j}(b)+\alpha\left(\delta^{i-1}(a)\right) \delta\left(r \delta^{j}(b)\right)=0$. Since $\delta^{i-1}(a) R \delta^{j}(b)=0$, we have $\alpha\left(\delta^{i-1}(a)\right) R \delta^{j}(b)=0$ by Lemma 3.1. Since $R$ is $(\alpha, \delta)$-weakly rigid, $\alpha\left(\delta^{i-1}(a)\right) \delta\left(r \delta^{j}(b)\right)=$ 0 for each $r \in R$. So $\delta^{i}(a) r \delta^{j}(b)=0$ for each $r \in R$, and so the result follows.

In [22, Proposition 3.2], the authors proved that a right semi-central idempotent $e$ of a ring $R$ is a right semi-central idempotent of $R[x ; \alpha, \delta]$ if and only if $e \in R \alpha(e)$.

COROLlary 3.3. If $R$ is an $\alpha$-weakly rigid ring, then each right semi-central idempotent of $R$ is a right semi-central idempotent of $R[x ; \alpha, \delta]$.

Proof. Let $e$ be a right semi-central idempotent of $R$. Then $e R=e R e$, so $e R(1-e)=0$. Since $R$ is $\alpha$-weakly rigid, $e \alpha(R(1-e))=0$, so $e=e \alpha(e)$. Now the result follows by [22, Proposition 3.2].

Recall that for a quasi-Baer ring $R$, for each left ideal $I$ of $R, \ell_{R}(I)=R e$ for some idempotent $e$ of $R$. Since $\ell_{R}(I)$ is an ideal, $e$ is right semi-central.

THEOREM 3.4. Let $R$ be an $\alpha$-weakly rigid ring. If $R$ is a quasi-Baer ring, then $R[x ; \alpha, \delta]$ is a quasi-Baer ring.

Proof. Let $I$ be an ideal of $S=R[x ; \alpha, \delta]$. Let $I_{0}$ be the set of all leading coefficients of elements of $I$ together with $0_{R}$. Then $I_{0}$ is a left ideal of $R$. So $\ell_{R}\left(I_{0}\right)=R e$ for some right semi-central idempotent $e$ of $R$. We prove that $\ell_{S}(I)=S e$. Let $f=a_{0}+\cdots+$ $a_{n} x^{n} \in I$ so $e f=e a_{0}+\cdots+e a_{n-1} x^{n-1} \in I$, since $e a_{n}=0$. So $e a_{n-1} \in I_{0}$; hence $e a_{n-1}=$ $e e a_{n-1}=0$. Continuing in this way it implies that $e a_{i}=0$ for each $0 \leq i \leq n$ and that ef $=0$. So $S e \subseteq \ell_{S}(I)$. Let $g=b_{0}+\cdots+b_{m} x^{m} \in \ell_{S}(I)$, so for each $f=a_{0}+\cdots+$ $a_{n} x^{n} \in I$ and $r \in R, g r f=0$. So $b_{m} \alpha^{m}\left(r a_{n}\right)=0$ for each $r \in R$, and hence $b_{m} \alpha^{m}\left(R a_{n}\right)=$ 0 . Since $R$ is $\alpha$-weakly rigid, $b_{m} R a_{n}=0$. So $b_{m} \in \ell_{R}\left(I_{0}\right)=R e$, and hence $b_{m}=b_{m} e$. On the other hand by Corollary 3.3, $e$ is a right semi-central idempotent of $S$. Thus $b_{m} x^{m}=$ $b_{m} e x^{m}=b_{m} e x^{m} e=b_{m} x^{m} e$, and hence $g r f=\left(b_{0}+\cdots+b_{m-1} x^{m-1}\right) r f+b_{m} x^{m}$ erf $=0$. But since $S e \subseteq \ell_{S}(I)$, we get $b_{m} x^{m} \operatorname{erf}=0$ so $\left(b_{0}+\cdots+b_{m-1} x^{m-1}\right) e f=0$. By a similar way we get $b_{m-1}=b_{m-1} e$ and that $b_{m-1} x^{m-1}=b_{m-1} x^{m-1} e$; so after $m$ steps, we can see that $b_{i}=b_{i} e$ and that $b_{i} x^{i}=b_{i} x^{i} e$ for each $i$. Thus $g=g e$, and hence $\ell_{S}(I) \subseteq S e$, and the result follows.

In [22, Example 2.1] the authors show that there exists a reduced Baer ring $R$ with a monomorphism $\alpha$ of $R$ such that $R[x ; \alpha]$ is not a p.q.-Baer ring. The example shows that $\alpha$-weakly rigid condition on $R$, in Theorem 3.4, is not superfluous.

In the proof of Lemma 3.5 and Theorem 3.6, we adapt the method which has been employed by Y. Zhou in [24]. 
Lemma 3.5. Let $R$ be an $(\alpha, \delta)$-weakly rigid ring. Let $L=\left\{\ell_{R}(R U) \mid U \subseteq R\right\}, M=$ $\left\{\ell_{S}(S U) \mid U \subseteq S=R[x ; \alpha, \delta]\right\}$ and $\Phi: L \rightarrow M$, given by $\Phi(I)=I[x ; \alpha, \delta]$ and $\Psi: M \rightarrow$ $L$, given by $\Psi(J)=J \cap R$; then $\Psi o \Phi=i d_{L}$.

Proof. We first show that, for $U \subseteq R, \ell_{R}(R U)[x ; \alpha, \delta]=\ell_{S}(S U)$. Let $f=a_{0}+\cdots+$ $a_{n} x^{n} \in \ell_{R}(R U)[x ; \alpha, \delta]$. So for each $i, a_{i} \in \ell_{R}(R U)$, and for each $u \in U, a_{i} R u=0$. So by Lemmas 3.1 and 3.2, $a_{i} R \alpha^{j}(u)=a_{i} R \delta^{j}(u)=0$ for each $j \geq 0$. Thus $f \in \ell_{S}(S U)$, and hence $\ell_{R}(R U)[x ; \alpha, \delta] \subseteq \ell_{S}(S U)$. Now assume that $g=b_{0}+\cdots+b_{m} x^{m} \in \ell_{S}(S U)$; then $\left(b_{0}+\cdots+b_{m} x^{m}\right) r u=0$ for each $r \in R$ and $u \in U$. So $b_{m} \alpha^{m}(r u)=0$, and hence $b_{m} \alpha^{m}(R u)=0$. Since $R$ is $\alpha$-weakly rigid, $b_{m} R u=0$, and hence $b_{m} \in \ell_{R}(R U)$. On the other hand $0=g r u=\left(b_{0}+\cdots+b_{m-1} x^{m-1}\right) r u+b_{m} x^{m} r u$. Since $b_{m} R u=0$, by Lemmas 3.1 and $3.2, b_{m} R \alpha^{j}(u)=b_{m} R \delta^{j}(u)=0$ for each $j \geq 0$. So $b_{m} x^{m} r u=0$; hence $\left(b_{0}+\cdots+\right.$ $\left.b_{m-1} x^{m-1}\right) r u=0$. By the same way we conclude that for each $i, b_{i} \in \ell_{R}(R U)$. Thus $g \in \ell_{R}(R U)[x ; \alpha, \delta]$, and hence $\ell_{R}(R U)[x ; \alpha, \delta]=\ell_{S}(S U)$. Therefore $\Phi$ is well defined. Next assume that $V \subseteq R[x ; \alpha, \delta]$. We show that $\ell_{S}(S V) \cap R=\ell_{R}\left(R C_{V}\right)$, where $C_{V}$ is the set of all coefficients of elements of $V$. If $f \in \ell_{S}(S V) \cap R$, then it is clear that $f \in \ell_{R}\left(R C_{V}\right)$. Let $a \in \ell_{R}\left(R C_{V}\right)$; then $a R b=0$ for each $b \in C_{V}$. So by Lemmas 3.1 and 3.2, $a R \alpha^{j}(b)=a R \delta^{j}(b)=0$ for each $j \geq 0$. So $a \in \ell_{S}(S V) \cap R$, whence $\ell_{S}(S V) \cap$ $R=\ell_{R}\left(R C_{V}\right)$, and $\Psi$ is well defined. Therefore $\Psi o \Phi\left(\ell_{R}(R U)\right)=\Psi\left(\ell_{R}(R U)[x ; \alpha, \delta]\right)=$ $\ell_{R}(R U)[x ; \alpha, \delta] \cap R=\ell_{S}(S U) \cap R=\ell_{R}\left(R C_{U}\right)=\ell_{R}(R U)$, and the result follows.

THEOREM 3.6. Let $R$ be an $(\alpha, \delta)$-weakly rigid ring. If $S=R[x ; \alpha, \delta]$ is quasi-Baer, then $R$ is quasi-Baer.

Proof. Let $I$ be an ideal of $R$. By Lemma 3.5, $\ell_{R}(I)[x ; \alpha, \delta]=\ell_{S}(S I)$. Since $S$ is quasi-Baer, for some idempotent $f=a_{0}+\cdots+a_{n} x^{n} \in S, \ell_{S}(S I)=S f$. But $\ell_{S}(S I)=$ $\ell_{R}(I)[x ; \alpha, \delta]$, so for each $0 \leq i \leq n, a_{i} \in \ell_{R}(I)$. On the other hand, by Lemma 3.5, $\ell_{R}(I)=\ell_{R}(I)[x ; \alpha, \delta] \cap R=\ell_{S}(S I) \cap R=S f \cap R$. So for each $a \in \ell_{R}(I), a=a f$, so $a=a a_{0}$. Since $a_{0} \in \ell_{R}(I), a_{0}=a_{0}^{2}$, and hence $\ell_{R}(I)=R a_{0}$, so the result follows.

COROLlaRY 3.7. Let $R$ be an $(\alpha, \delta)$-weakly rigid ring. Then, $R$ is quasi-Baer if and only if $R[x ; \alpha, \delta]$ is quasi-Baer.

In [21, Example 3.6] the authors provided some examples of quasi-Baer rings $R[x ; \delta]$, such that $R$ is not p.q.-Baer. So the condition $\delta$-weakly rigid in Theorem 3.6 is not superfluous.

The following (see [14, Example 9]) is an example of a ring $R$ such that $R[x ; \alpha]$ is quasi-Baer, but $R$ is not p.q.-Baer, so the condition $\alpha$-weakly rigid in Theorem 3.6 is not superfluous.

EXAMPLE 3.8. Let $R=\{(a, b) \in \mathbb{Z} \oplus \mathbb{Z} \mid a \equiv b \bmod 2\} ; \alpha: R \rightarrow R$ given by $\alpha(a, b)=(b, a)$. Then by [14, Example 9], $R[x ; \alpha]$ is quasi-Baer, but $R$ is not p.q.-Baer.

THEOREM 3.9. Let $R$ be an $\alpha$-weakly rigid ring. If $R$ is a left p.q.-Baer ring, then $R[x ; \alpha, \delta]$ is a left p.q.-Baer ring.

Proof. Let $R$ be a left p.q.-Baer $\alpha$-weakly rigid ring. Let $f=a_{0}+\cdots+a_{n} x^{n} \in S=$ $R[x ; \alpha, \delta]$. For each $0 \leq i \leq n$ we have $\ell_{R}\left(R a_{i}\right)=R e_{i}$ for some right semi-central idempotent $e_{i} \in R$. Put $e=e_{n} e_{n-1} \cdots e_{0}$. Since for each $0 \leq i \leq n, e_{i}$ is a right semi-central idempotent of $R$, we get $e^{2}=e_{n} e_{n-1} \cdots e_{0} e_{n} e_{n-1} \cdots e_{0}=e_{n} e_{n-1} \cdots e_{0} e_{n-1} e_{n-2} \cdots e_{0}=$ $e_{n} e_{n-1} \cdots e_{0} e_{n-2} \cdots e_{0}=\cdots=e_{n} e_{n-1} \cdots e_{0}=e$. Now for each $r \in R$ we have $e r=$ $e_{n} e_{n-1} \cdots e_{0} r e_{n}=e_{n} e_{n-1} \cdots e_{0} r e_{n} e_{n-1}=\cdots=e_{n} e_{n-1} \cdots e_{0} r e_{n} e_{n-1} \cdots e_{0}=$ ere. Thus $e$ 
is a right semi-central idempotent of $R$. By Corollary 3.3, $e$ is a right semicentral idempotent in $S$. Now we claim that $\ell_{S}(S f)=S e$. Since $e$ is right semicentral in $S$, we have $e S f=e S e f=0$. So $S e \subseteq \ell_{S}(S f)$. Now suppose that $g=$ $b_{0}+b_{1} x+\cdots+b_{m} x^{m} \in \ell_{S}(S f)$, so $g R f=0$, and hence $b_{m} \alpha^{m}\left(R a_{n}\right)=0$. Since $R$ is $\alpha$-weakly rigid, $b_{m} R a_{n}=0$, and hence $b_{m} \in \ell_{R}\left(R a_{n}\right)$. Thus $b_{m}=b_{m} e_{n}$. Since $g S f=$ $0, g e_{n} R f=0$. But $e_{n} R=e_{n} R e_{n}$, so $g e_{n} \operatorname{Re}_{n}\left(a_{0}+\cdots+a_{n} x^{n}\right)=0$. So $g e_{n} \operatorname{Re}_{n}\left(a_{0}+\right.$ $\left.\cdots+a_{n-1} x^{n-1}\right)=0$. Thus $b_{m} \alpha^{m}\left(e_{n} R a_{n-1}\right)=0$. On the other hand $e_{n} R=e_{n} R e_{n}$ so $e_{n} R\left(1-e_{n}\right)=0$; hence $e_{n} \alpha\left(1-e_{n}\right)=0$, since $R$ is $\alpha$-weakly rigid. Thus $e_{n}=e_{n} \alpha\left(e_{n}\right)$. So $e_{n}=e_{n} \alpha\left(e_{n} \alpha\left(e_{n}\right)\right)=e_{n} \alpha\left(e_{n}\right) \alpha^{2}\left(e_{n}\right)=e_{n} \alpha^{2}\left(e_{n}\right)$. Inductively we can see that $e_{n}=e_{n} \alpha^{t}\left(e_{n}\right)$ for each positive integer $t$. Hence $0=b_{m} \alpha^{m}\left(e_{n} R a_{n-1}\right)=b_{m} e_{n} \alpha^{m}\left(e_{n}\right) \alpha^{m}\left(R a_{n-1}\right)=$ $b_{m} e_{n} \alpha^{m}\left(R a_{n-1}\right)=b_{m} \alpha^{m}\left(R a_{n-1}\right)$. So we get $b_{m} R a_{n-1}=0$, and hence $b_{m}=b_{m} e_{n-1}=$ $b_{m} e_{n} e_{n-1}$. After repeating this argument $n$ times we get $b_{m}=b_{m} e_{n} e_{n-1} \cdots e_{0}=b_{m} e$. Since $g S f=0$, we deduce that $\left(b_{0}+b_{1} x+\cdots+b_{m} x^{m}\right) S f=0$, so $\left(b_{0}+b_{1} x+\cdots+\right.$ $\left.b_{m-1} x^{m-1}\right) S f+b_{m} x^{m} S f=0$. But $b_{m}=b_{m} e$ so $b_{m} x^{m} S f=b_{m} e x^{m} S f=b_{m} e x^{m} S e f=0$. So $\left(b_{0}+b_{1} x+\cdots+b_{m-1} x^{m-1}\right) S f=0$. By the same method we see that $b_{m-1}=b_{m-1} e$. After repeating this argument $m$ times, we see that $b_{i}=b_{i} e$ for each $0 \leq i \leq m$. Therefore $g=g e$, and the result follows.

Note that [22, Example 2.1] shows that the $\alpha$-weakly rigid condition in Theorem 3.9 is not superfluous.

Corollary 3.10. If $R$ is a left p.q.-Baer ring with a derivation $\delta$, then $R[x ; \delta]$ is a left p.q.-Baer ring.

THEOREM 3.11. Let $R$ be an $(\alpha, \delta)$-weakly rigid ring. If $R[x ; \alpha, \delta]$ is left p.q.-Baer, then $R$ is left p.q.-Baer.

Proof. The proof is similar to that of Theorem 3.6.

COROLlary 3.12. Let $R$ be an $(\alpha, \delta)$-weakly rigid ring. Then $R$ is left p.q.-Baer if and only if $R[x ; \alpha, \delta]$ is left p.q.-Baer.

Example 3.8 and [21, Example 3.6] show that the condition $(\alpha, \delta)$-weakly rigid in Theorem 3.11 is not superfluous.

The set $\left\{x^{i}\right\}_{i \geq 0}$ is easily seen to be a left Ore subset of $S=R[x ; \alpha]$, so that one can localise $S$ and form the skew Laurent polynomial ring $T=R\left[x, x^{-1} ; \alpha\right]$. Elements of $T$ are finite sums of elements of the form $x^{-j} r x^{i}$, where $r \in R$ and $i, j$ are non-negative integers. Multiplication is subject to $x r=\alpha(r) x$ for all $r \in R$. In the case in which $\alpha$ is an automorphism; elements of $T$ have the form $\Sigma_{i=m}^{n} r_{i} x^{i}$, where $r_{i} \in R$ and $m, n \in \mathbb{Z}$. We consider D. A. Jordan's [16] construction of the $\operatorname{ring} A(R, \alpha)$. Let $A(R, \alpha)$ or $A$ be the subset $\left\{x^{-i} r x^{i} \mid r \in R, i \geq 0\right\}$ of the skew Laurent polynomial ring $R\left[x, x^{-1} ; \alpha\right]$. For each $j \geq 0, x^{-i} r x^{i}=x^{-(i+j)} \alpha^{j}(r) x^{(i+j)}$. It follows that the set of all such elements forms a subring of $R\left[x, x^{-1} ; \alpha\right]$ with

$$
x^{-i} r x^{i}+x^{-j} s x^{j}=x^{-(i+j)}\left(\alpha^{j}(r)+\alpha^{i}(s)\right) x^{(i+j)}
$$

and

$$
\left(x^{-i} r x^{i}\right)\left(x^{-j} s x^{j}\right)=x^{-(i+j)} \alpha^{j}(r) \alpha^{i}(s) x^{(i+j)}
$$

for $r, s \in R$ and $i, j \geq 0$. Note that $\alpha: A(R, \alpha) \rightarrow A(R, \alpha)$, given by $\alpha\left(x^{-i} r x^{i}\right)=$ $x^{-i} \alpha(r) x^{i}$, is actually an automorphism of $A(R, \alpha)$; this is because $x^{-i} r x^{i}=$ $\alpha\left(x^{-(i+1)} r x^{i+1}\right)$, for each $i \geq 0$ and each $r \in R$. We have $R\left[x, x^{-1} ; \alpha\right] \simeq A\left[x, x^{-1} ; \alpha\right]$, 
by way of an isomorphism which maps $x^{-i} r x^{j}$ to $\alpha^{-i}(r) x^{j-i}$, for each $i, j$, (See [16], for more details).

PROPOSITION 3.13. Let $R$ be an $\alpha$-weakly rigid ring. Then $R$ is quasi-Baer if and only if $A(R, \alpha)$ is quasi-Baer.

Proof. Let $R$ be a quasi-Baer ring and $I$ an ideal of $A=A(R, \alpha)$. Put $B=\{a \in$ $R \mid x^{-i} a x^{i} \in I$ for some $\left.i \geq 0\right\}$. Let $J=R B R$; then $\ell_{R}(J)=R e$ for some right semicentral idempotent $e$ of $R$. Now we show that $\ell_{A}(I)=A e$. Since $e R(1-e)=0$ and $R$ is $\alpha$-weakly rigid, $\alpha(e) R(1-e)=0$ so $\alpha(e)=\alpha(e) e$. So for each $i>0, \alpha^{i}(e)=$ $\alpha^{i}(e) \alpha^{i-1}(e) \ldots \alpha(e) e$. Hence for each $x^{-i} a x^{i} \in I$, we have $e x^{-i} a x^{i}=x^{-i} \alpha^{i}(e) a x^{i}=$ $x^{-i} \alpha^{i}(e) \alpha^{i-1}(e) \ldots e a x^{i}=0$, which implies that $A e \subseteq \ell_{A}(I)$. Now if $x^{-i} a x^{i} \in \ell_{A}(I)$, then for each $x^{-t} b x^{t} \in I$ and $r \in R, x^{-i} a x^{i} r x^{-t} b x^{t}=0$. So $x^{-(i+t)} \alpha^{t}(a) \alpha^{i}(r b) x^{i+t}=0$. Hence $\alpha^{t}(a) \alpha^{i}(R b)=0$. Since $R$ is $\alpha$-weakly rigid, $\alpha^{t}(a) R b=0$, and hence $a R b=0$ by Lemma 3.1. So $a \in \ell_{R}(J)$, and hence $a=a e$; thus $x^{-i} a x^{i}=x^{-i} a e x^{i}$. On the other hand since $e R(1-e)=0$ and $R$ is $\alpha$-weakly rigid, $e \alpha^{i}(R(1-e))=0$, so $e=e \alpha^{i}(e)$. Thus $x^{-i} a x^{i}=x^{-i} a e x^{i}=x^{-i} a e \alpha^{i}(e) x^{i}=x^{-i} a e x^{i} e=x^{-i} a x^{i} e$. Therefore $\ell_{A}(I) \subseteq A e$, and the result follows. Conversely suppose that $A(R, \alpha)$ is a quasi-Baer ring and $I$ an ideal of $R$. So $r_{A}(I A)=e A$ for some idempotent $e \in A$. Let $e=x^{-i} a x^{i}$, where $a=a^{2} \in R$. We now show that $r_{R}(I)=a R$. Since $I A e=0$, for each $r \in R$ and $b \in I, b x^{-i} r x^{i} x^{-i} a x^{i}=0$. So $x^{-i} \alpha^{i}(b) r a x^{i}=0$, and hence $\alpha^{i}(b) R a=0$. Since $R$ is $\alpha$ weakly rigid, $b R a=0$, so $a R \subseteq r_{R}(I)$. Now if $b \in r_{R}(I)$, then for each $c \in I$ and $x^{-j} r x^{j} \in A, c x^{-j} r x^{j} x^{-i} b x^{i}=x^{-(i+j)} \alpha^{i+j}(c) \alpha^{i}(r) \alpha^{j}(b) x^{i+j}$. On the other hand $c R b=0$ so $\alpha^{i+j}(c) R \alpha^{j}(b)=0$. So $\alpha^{i+j}(c) \alpha^{i}(r) \alpha^{j}(b)=0$, and hence $x^{-i} b x^{i} \in r_{A}(I A)=e A$. So $x^{-i} b x^{i}=x^{-i} a x^{i} x^{-i} b x^{i}$, and hence $b=a b$, and the result follows.

THEOREM 3.14. Let $R$ be an $\alpha$-weakly rigid ring and $\alpha$ an automorphism of $R$. If $R$ is quasi-Baer, then $R\left[x, x^{-1} ; \alpha\right]$ is quasi-Baer.

Proof. Since $\alpha$ is an automorphism of $R$, each element of $R\left[x, x^{-1} ; \alpha\right]$ is of the form $\sum_{i=m}^{n} r_{i} x^{i}$, where $r_{i} \in R$ and $m, n \in \mathbb{Z}$, so the proof is similar to that of Theorem 3.4 .

THEOREM 3.15. Let $R$ be an $\alpha$-weakly rigid ring. If $R$ is a quasi-Baer ring, then $R\left[x, x^{-1} ; \alpha\right]$ is a quasi-Baer ring.

Proof. Since $R$ is $\alpha$-weakly rigid quasi-Baer, $A$ is quasi-Baer. Since $\alpha$ is an automorphism of $A$ and $R\left[x, x^{-1} ; \alpha\right] \simeq A\left[x, x^{-1} ; \alpha\right]$, so the result follows by Theorem 3.14 .

LEMMA 3.16. Let $R$ be an $\alpha$-weakly rigid ring and $\alpha$ an automorphism of $R$. Let $L=\left\{\ell_{R}(R U) \mid U \subseteq R\right\}, M=\left\{\ell_{S}(S U) \mid U \subseteq S=R\left[x, x^{-1} ; \alpha\right]\right\}$ and $\Phi: L \rightarrow M$, given by $\Phi(I)=I\left[x, x^{-1} ; \alpha\right]$ and $\Psi: M \rightarrow L$, given by $\Psi(J)=J \cap R$; then $\Psi o \Phi=i d_{L}$.

Proof. The proof is similar to that of Lemma 3.5.

THEOREM 3.17. Let $R$ be an $\alpha$-weakly rigid ring and $\alpha$ an automorphism of $R$. If $R\left[x, x^{-1} ; \alpha\right]$ is quasi-Baer, then $R$ is quasi-Baer.

Proof. The proof is similar to that of Theorem 3.6.

Proposition 3.18. If $R$ is an $\alpha$-weakly rigid ring, then $A(R, \alpha)$ is an $\alpha$-weakly rigid ring. 
Proof. The proof is easy.

THEOREM 3.19. Let $R$ be an $\alpha$-weakly rigid ring. If $R\left[x, x^{-1} ; \alpha\right]$ is quasi-Baer, then $R$ is quasi-Baer.

Proof. Since $R$ is $\alpha$-weakly rigid, $A$ is $\alpha$-weakly rigid by proposition 3.18. Since $\alpha$ is an automorphism of $A$ and $R\left[x, x^{-1} ; \alpha\right] \simeq A\left[x, x^{-1} ; \alpha\right]$, by Theorem $3.18, A$ is quasi-Baer, and the result follows by Proposition 3.13.

COROLlary 3.20. Let $R$ be an $\alpha$-weakly rigid ring. Then $R$ is quasi-Baer if and only if $R\left[x, x^{-1} ; \alpha\right]$ is quasi-Baer.

THEOREM 3.21. Let $R$ be an $\alpha$-weakly rigid ring and $\alpha$ an automorphism of $R$. If $R$ is a left p.q.-Baer ring, then $R\left[x, x^{-1} ; \alpha\right]$ is a left p.q.-Baer ring.

Proof. Let $f \in S=R\left[x, x^{-1} ; \alpha\right]$. Since $\alpha$ is an automorphism, $f=x^{-m} a_{-m}+$ $x^{-m+1} a_{-m+1}+\cdots+a_{0}+a_{1} x+\cdots+a_{n} x^{n} . \quad$ Set $\quad J=R a_{-m}+R a_{-m+1}+\cdots+$ $R a_{0}+\cdots+R a_{n}$, which is a left ideal of $R$, so $\ell_{R}(J)=R e$, by [6, Proposition 1.7]. By a similar method as in the proof of Theorem 3.4, we can show that $\ell_{S}(S f)=S e$, and the result follows.

Proposition 3.22. Let $R$ be an $\alpha$-weakly rigid ring. Then $R$ is a left p.q.-Baer ring if and only if $A(R, \alpha)$ is a left p.q.-Baer ring.

Proof. The proof is similar to that of Proposition 3.13.

THEOREM 3.23. Let $R$ be an $\alpha$-weakly rigid ring with an automorphism $\alpha$. If $R\left[x, x^{-1} ; \alpha\right]$ is a left p.q.-Baer ring, then $R$ is left p.q.-Baer.

Proof. Using Lemma 3.16, the proof is similar to that of Theorem 3.6.

THEOREM 3.24. Let $R$ be an $\alpha$-weakly rigid ring. Then $R$ is a left p.q.-Baer ring if and only if $R\left[x, x^{-1} ; \alpha\right]$ is a left p.q.-Baer ring.

Proof. Since $R\left[x, x^{-1} ; \alpha\right] \simeq A\left[x, x^{-1} ; \alpha\right]$ and $\alpha$ is an automorphism of $A$, the result follows using Theorems 3.21 and 3.23 and Proposition 3.22.

The following (see [12, Example 3.6]) is an example of a $\operatorname{ring} R$ such that $R\left[x, x^{-1} ; \alpha\right]$ is quasi-Baer, but $R$ is not p.q.-Baer, so the condition $\alpha$-weakly rigid in Corollary 3.20 and Theorem 3.24 is not superfluous.

ExAMPLE 3.25. Let $R$ and $\alpha$ be those given in Example 3.8. Then by [12, Example 3.6], $R\left[x, x^{-1} ; \alpha\right]$ is quasi-Baer, but $R$ is not p.q.-Baer.

COROLlARY 3.26. Let $R$ be an $(\alpha, \delta)$-weakly rigid ring, $\alpha$ an endomorphism and $\delta$ an $\alpha$-derivation of $R$. Then the following are equivalent:

(i) $R$ is a left p.q.-Baer ring;

(ii) $A(R, \alpha)$ is a left p.q.-Baer ring;

(iii) $R[x ; \alpha, \delta]$ is a left p.q.-Baer ring;

(iv) $R\left[x, x^{-1} ; \alpha\right]$ is a left p.q.-Baer ring. 
COROLlary 3.27 [7, Theorem 3.1]. The following are equivalent:

(1) $R$ is a left p.q.-Baer ring;

(2) $R[x]$ is a left p.q.-Baer ring;

(3) $R\left[x, x^{-1}\right]$ is a left p.q.-Baer ring.

THEOREM 3.28. Let $R$ be a $\alpha$-weakly rigid ring. If $R$ is quasi-Baer, then $R[[x ; \alpha]]$ is quasi-Baer.

Proof. Let $I$ be an ideal of $S=R[[x ; \alpha]]$. Let $J=\left\{a \in R \mid\right.$ there exists $a x^{m}+$ $a_{m+1} x^{m+1}+\cdots \in I$, for some non-negative integer $m$ and $\left.a_{i} \in R\right\}$. Then $J$ is a left ideal of $R$. So $\ell_{R}(J)=R e$ for some right semi-central idempotent $e$ of $R$. We show that $\ell_{S}(I)=S e$. If $f=\sum_{i=m}^{\infty} a_{i} x^{i} \in I$, then $a_{m} \in J$, so $e a_{m}=0$. Hence $e f=e a_{m+1} x^{m+1}+$ $e a_{m+2} x^{m+2}+\cdots$. Since $e a_{m+1} \in J$, we have $e a_{m+1}=e e a_{m+1}=0$. By this way we get $e f=0$ so $S e \subseteq \ell_{S}(I)$. Now assume $g=\sum_{j=n}^{\infty} b_{j} x^{j} \in \ell_{S}(I)$, so for each $f=\sum_{i=m}^{\infty} a_{i} x^{i} \in$ $I$ and $r \in R, g r f=0$. So $b_{n} \alpha^{n}\left(r a_{m}\right)=0$ for each $r \in R$, and hence $b_{n} \alpha^{n}\left(R a_{m}\right)=0$. So $b_{n} R a_{m}=0$; hence $b_{n} \in \ell_{R}(J)$, so $b_{n}=b_{n}$. Thus $g r f=b_{n} x^{n} r f+\sum_{j=n+1}^{\infty} b_{j} x^{j} r f=0$. Since $e$ is right semi-central, $e R=e R e$ so $e R(1-e)=0$. Since $R$ is $\alpha$-weakly rigid, $e \alpha^{n}(R(1-e))=0$ for each positive integer $n$. So $e=e \alpha^{n}(e)$, and hence $b_{n} x^{n} r f=$ $b_{n} e x^{n} r f=b_{n} e \alpha^{n}(e) x^{n} r f=b_{n} e x^{n}$ erf. But we have erf $=0$, so $b_{n} x^{n} r f=0$, and hence $\sum_{j=n+1}^{\infty} b_{j} x^{j} r f=0$. By the same way we can see that $b_{n+1}=b_{n+1} e$ and by induction for each $i$ that $b_{i}=b_{i} e$, so $g=\sum_{j=n}^{\infty} b_{j} e x^{j}$. On the other hand, for each $j, e=e \alpha^{j}(e)$, so $g=\sum_{j=n}^{\infty} b_{j} e x^{j} e=\sum_{j=n}^{\infty} b_{j} x^{j} e=g e$. So the result follows.

LEMMA 3.29. Let $R$ be an $\alpha$-weakly rigid ring. Let $L=\left\{\ell_{R}(R U) \mid U \subseteq R\right\}, M=$ $\left\{\ell_{S}(S U) \mid U \subseteq S=R[[x ; \alpha]]\right\}$ and $\Phi: L \rightarrow M$, given by $\Phi(I)=I[[x ; \alpha]]$, and $\Psi: M \rightarrow$ $L$, given by $\Psi(J)=J \cap R$; then $\Psi o \Phi=i d_{L}$.

Proof. The proof is similar to that of Lemma 3.5.

THEOREM 3.30. Let $R$ be an $\alpha$-weakly rigid ring. If $R[[x ; \alpha]]$ is quasi-Baer, then $R$ is quasi-Baer.

Proof. The proof is similar to that of Theorem 3.6.

COROLlARY 3.31. Let $R$ be an $(\alpha, \delta)$-weakly rigid ring, $\alpha$ an endomorphism and $\delta$ an $\alpha$-derivation of $R$. Then the following are equivalent:

(i) $R$ is a quasi-Baer ring;

(ii) $A(R, \alpha)$ is a quasi-Baer ring;

(iii) $R[x ; \alpha, \delta]$ is a quasi-Baer ring;

(iv) $R\left[x, x^{-1} ; \alpha\right]$ is a quasi-Baer ring;

(v) $R[[x ; \alpha]]$ is a quasi-Baer ring.

THEOREM 3.32. Let $R$ be an $\alpha$-weakly rigid ring, with $\alpha$ an automorphism of $R$. If $R$ is quasi-Baer, then $R\left[\left[x, x^{-1} ; \alpha\right]\right]$ is quasi-Baer.

Proof. Since $\alpha$ is an automorphism of $R$, the elements of $R\left[\left[x, x^{-1} ; \alpha\right]\right]$ can be written in the form $a_{-m} x^{-m}+\cdots+a_{0}+a_{1} x+\cdots$, where $m$ is a positive integer and $a_{i} \in R$ for each $i$. So the proof is similar to that of 3.28 .

LEMMA 3.33. Let $R$ be an $\alpha$-weakly rigid ring and $\alpha$ an automorphism of $R$. Let $L=$ $\left\{\ell_{R}(R U) \mid U \subseteq R\right\}, M=\left\{\ell_{S}(S U) \mid U \subseteq S=R\left[\left[x, x^{-1} ; \alpha\right]\right]\right\}$ and $\Phi: L \rightarrow M$, given by $\Phi(I)=I\left[\left[x, x^{-1} ; \alpha\right]\right]$, and $\Psi: M \rightarrow L$, given by $\Psi(J)=J \cap R$; then $\Psi o \Phi=i d_{L}$.

Proof. The proof is similar to that of Lemma 3.5. 
THEOREM 3.34. Let $R$ be an $\alpha$-weakly rigid ring and $\alpha$ an automorphism of $R$. If $R\left[\left[x, x^{-1} ; \alpha\right]\right]$ is quasi-Baer, then $R$ is quasi-Baer.

Proof. The proof is similar to that of Theorem 3.6.

COROLlary 3.35. Let $R$ be an $\alpha$-weakly rigid ring and $\alpha$ an automorphism of $R$. Then $R$ is quasi-Baer if and only if $R\left[\left[x, x^{-1} ; \alpha\right]\right]$ is quasi-Baer.

COROLlary 3.36. [5, Theorem 1.8] The following are equivalent.

(i) $R$ is a quasi-Baer ring;

(ii) $R[x]$ is a quasi-Baer ring;

(iii) $R[[x]]$ is a quasi-Baer ring;

(iv) $R\left[x, x^{-1}\right]$ is a quasi-Baer ring;

(v) $R\left[\left[x, x^{-1}\right]\right]$ is a quasi-Baer ring.

Notice that, Birkenmeier et al.'s proof of [5, Lemma 1.7] to show that either $R\left[x ; x^{-1}\right]$ or $R\left[\left[x ; x^{-1}\right]\right]$ is quasi-Baer implies $R$ is quasi-Baer involves a long and quite technical calculation.

ACKNOWLEDGEMENTS. The authors are indebted to the referee for various valuable comments and suggestions for the improvement of this paper.

\section{REFERENCES}

1. E. P. Armendariz, A note on extensions of Baer and p.p.-rings, J. Austral. Math. Soc. 18 (1974), 470-473.

2. S. K. Berberian, Baer *-rings, (Springer, Berlin, 1972).

3. G. F. Birkenmeier, Baer rings and quasi-continuous rings have a MDSN, Pacific J. Math. 97 (1981), 283-292. $319-326$.

4. G. F. Birkenmeier, Decompositions of Baer-like rings, Acta Math. Hungar. 59 (1992),

5. G. F. Birkenmeier, J. Y. Kim and J. K. Park, Polynomial extensions of Baer and quasiBaer rings, J. Pure Appl. Algebra 159 (2001), 24-42.

6. G. F. Birkenmeier, J. Y. Kim and J. K. Park, Principally quasi-Baer rings, Comm. Algebra 29(2) (2001), 639-660.

7. G. F. Birkenmeier, J. Y. Kim and J. K. Park, On polynomial extensions of principally quasi-Baer rings, Kyungpook Math. J. 40 (2000), 247-253.

8. G. F. Birkenmeier, J. K. Park and S. T. Rizvi, Ring hulls and applications, J. Algebra 304(2) (2006), 633-665.

9. W. E. Clark, Twisted matrix units semigroup algebras, Duke Math. J. 34 (1967), 417-424.

10. C. Faith, Injective quotient rings of commutative rings, in Module Theory, Lecture Notes in Mathematics 700, (Proc. Special Session, Amer. Math. Soc., Univ. Washington, Seattle, Wash., 1977) (Springer, Berlin), 151-203.

11. J. Han, Y. Hirano and H. Kim, Semiprime ore extensions, Comm. Algebra 28(8) (2000) 3795-3801.

12. E. Hashemi and A. Moussavi, Polynomial extensions of quasi-Baer rings. Acta Math. Hungar. 107(3) (2005), 207-224.

13. Y. Hirano, On annihilator ideals of a polynomial ring over a noncommutative ring, J. Pure Appl. Algebra 168 (2002), 45-52.

14. C. Y. Hong, N. K. Kim, T. Kwak, Ore extensions of Baer and p.p.-rings, J. Pure Appl. Algebra 151 (2000), 215-226.

15. C. Y. Hong, N. K. Kim, T. Kwak and S. Tariq Rizvi, Rigid ideals and radicals of Ore extensions, Algebra Colloq. 12(3) (2005), 399-412.

16. D. A. Jordan, Bijective extensions of injective ring endomorphisms, J. Lond. Math. Soc. 25(2) (1982), 435-448.

17. I. Kaplansky, Rings of operators, (Benjamin, New York, 1965). 
18. J. Krempa, Some examples of reduced rings, Algebra Colloq. 3(4) (1996) 289-300.

19. T. K. Lee and Y. Zhou, Armendariz and reduced rings, Comm. Algebra 32(6) (2004), 2287-2299.

20. Y. Lee and C. Huh, Counterexamples on p.p.-rings, Kyungpook Math. J. 38 (1998), 421-427.

21. A. R. Nasr-Isfahani and A. Moussavi, Baer and quasi-Baer differential polynomial rings, Comm. Algebra 36(9) (2008), 3533-3542.

22. A. R. Nasr-Isfahani and A. Moussavi, Ore extensions of quasi-Baer rings, J. Algebra Appl. 7(2) (2008), 211-224.

23. P. Pollingher and A. Zaks, On Baer and quasi-Baer rings, Duke Math. J. 37 (1970), $127-138$.

24. Y. Zhou, A simple proof of a theorem on quasi-Baer rings, Arch. Math. 81 (2003), 253-254. 